\title{
Designing Multi-Stage Coupled Convex Programming with Data-Driven McCormick Envelope Relaxations for Motion Planning
}

\author{
Xuan $\operatorname{Lin}^{1 *}$, Min Sung $\mathrm{Ahn}^{1 *}$, and Dennis Hong ${ }^{1}$
}

\begin{abstract}
For multi-limbed robots, motion planning with posture and force constraints tends to be a difficult optimization problem due to nonlinearities, which also present extended solve times. We propose a multi-stage optimization framework with data-driven inter-stage coupling constraints to address the nonlinearity. Both clustering and evolutionary approaches to find the McCormick envelope relaxations are used to find the problem-specific parameters. The learned constraints are then used in the prior stages, which provides advanced knowledge of the following stages. This leads to improved solve times and interpretability of the results. The planner is validated through multiple walking and climbing tasks on a $10 \mathrm{~kg}$ hexapod robot.
\end{abstract}

\section{INTRODUCTION}

Legged robots present a unique advantage compared to their wheeled counterparts, where discrete contact with the environment can be made to maneuver discontinuous, complex terrains. To fully realize this capability, they often use motion planning to autonomously choose their footholds and plan their body movements to avoid slipping and tumbling. Optimization-based techniques have been exploited by researchers to resolve the motion planning problem [1][4]. The easiest way to implement optimization for motion planning would be to include all the linear or nonlinear equations into a single optimization problem. Unfortunately, such an approach often suffers from time complexity [2], although recent efforts to parallelize optimizations have shown speedups [5]. To reduce the solving time without such techniques, one approach is to separate the entire problem into multiple stages, where each stage only solves for a subset of the decision variables [6]-[9]. However, if each stage independently finds solutions without considering the subsequent stages' constraints, the later stages may result in no feasible solution. This issue exacerbates when the problem scales to many stages.

This paper proposes a multi-staged trajectory optimization (TO) formulation with inter-stage couplings learned from data. We relax the nonlinear constraints in the later stages into convex forms, and embed them into the prior stages, resulting in the first stage solving an approximated form of the original problem and subsequent stages projecting the solutions back to the nonlinear manifolds to correct the error. While dealing with the TO problem as a whole is complicated, the clear interpretability of the convex optimization permits us to segment the constraints out, perform dedicated

${ }^{1}$ X. Lin, M. S. Ahn, and D. Hong are with the Robotics and Mechanisms Laboratory, Department of Mechanical and Aerospace Engineering, University of California, Los Angeles, CA 90095, USA. \{maynight, aminsung, dennishong\} @ucla.edu

*X. Lin and M. S. Ahn contributed equally to this work.

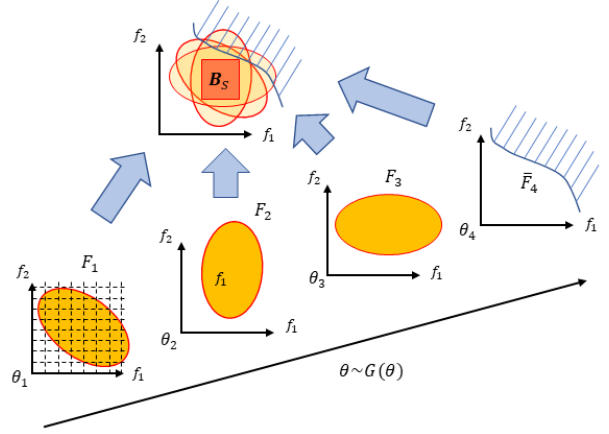

Fig. 1: The proposed approach to find $\mathbf{B}_{\mathcal{F}}$ inside the intersection of solution set $F_{i}$ 's and exclude infeasible solution set $\bar{F}_{i}$ 's. While we can finely grid the variable space and accurately approximate $F_{i}$ 's in each grid (shown in $F_{1}$ plot), the proposed approach results in less integer variables.

learning, and place them back into the structure. Learning is done using both clustering and genetic algorithm (GA), where we identify the regions that the data populate and fit constraints around that region. The resultant formulation is tailored to the given type of problem, and solve time and interpretability are improved.

While the proposed method is applicable for general constraints, we tackle a simple nonlinear constraint-the bilinear constraint—by embedding it as a McCormick envelope in the first optimization stage. McCormick envelopes are the best linear relaxation for bilinear constraints [10]. They are widely used to formulate bilinear constraints into mixed-integer convex programming (MICPs) [11], where the complete bilinear surface is divided evenly into multiple envelopes. This way of segmentation is general but usually results in too many integer variables and extended solving time. To reduce solve time, previous studies learned optimal strategies with neural networks to provide the best choice of integer variables to the MICP solver [12], [13]. However, since the number of strategies is an exponential function of the number of integer variables, the number of class labels quickly becomes intractable as the problem gets more complicated. It may be more efficient to directly conduct learning on the MICP framework to reduce the number of integer variables before it is handed to any learning agent. Instead of evenly dividing the bilinear surface into envelopes, we propose to adapt the placement of envelopes to the given type of problems, resulting in a reduced number of integer variables. Adaptive envelope tuning can be seen in mixed-integer nonlinear programming (MINLP) [14]-[16] where the general idea is to fit envelopes to the more promising regions on the solution manifold as the problem is being solved. Our method makes use of learning to perform adaptation before 


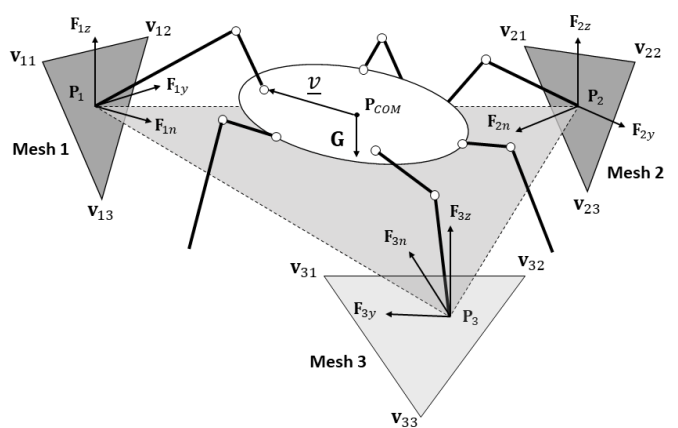

Fig. 2: A robot making 3 contacts with the environment, subject to gravity. Contact planes are represented by triangular meshes with vertices $\mathbf{v}_{s j}(i, j=$ $1,2,3) . \mathbf{F}_{s n}, \mathbf{F}_{s y}, \mathbf{F}_{s z}$ are constant vectors along the normal and two predefined shear directions. The three contact points form a contact triangle.

the solving process is started. In this paper, we focus on solving the motion planning problem applied to multi-legged robot locomotion on multiple terrains. Hardware testings are done to show feasibility. Our contributions are:

1) A multi-stage coupled optimization-based motion planning framework with the later stages relaxed and embedded into the earlier stages.

2) Learning best McCormick envelope relaxation with clustering and GA techniques from data.

3) Demonstration of the planning results on hardware.

\section{PRoblem SETUP}

This section presents the formulations of the motion planning problem which is to be ultimately solved.

A multi-limbed robot is assumed to make $N$ point contacts (i.e. pure contact force, no contact moment) with the environment. We denote the contact points with index $i$ where $i=$ $1, \ldots, N$. We model the environment with polygon meshes ( e.g. a triangular mesh is depicted in Fig. 2). Additionally, we assume that mesh $i$ 's vertices (corresponding to the limb $i$ in contact with the mesh) are denoted by $\mathbf{v}_{i u}$, where $u$ is the indices of the vertices, $u=1, \ldots, U$ and $U$ is the number of vertices for mesh $i$. The normal direction $\mathbf{n}_{i}$ can be retrieved via a perception system. If we define $\mathbf{p}_{i}^{w}$ to be the position of toe $i$ within mesh $i$ with respect to the world frame:

$$
\mathbf{p}_{i}^{w}=\sum_{u} p_{i u}^{w} \mathbf{v}_{i u}, \sum_{j} p_{i u}^{w}=1, p_{i u}^{w} \in[0,1]
$$

If we define $\mathbf{p}_{i}^{b}$ to be the position of toe $i$ with respect to the origin of the body coordinate system, we have:

$$
\mathbf{p}_{i}^{w}=\mathbf{p}_{C O M}+\mathbf{p}_{i}^{b}
$$

where $\mathbf{p}_{C O M}$ is the position of the center of mass (COM) with respect to the origin of the global coordinate system.

Let the contact force on limb $i$ be denoted by $\mathbf{f}_{i}$. Since $\mathbf{n}_{i}$ is known, we can define two mutually perpendicular shear directions $\mathbf{y}_{i}$ and $\mathbf{z}_{i}$, which can be pre-defined for every mesh. The three directional vectors form a local mesh coordinate frame, and the contact force can be represented as $\mathbf{f}_{i}=\mathbf{f}_{i n}+\mathbf{f}_{i y}+\mathbf{f}_{i z}$ in the mesh frame, where $\mathbf{f}_{i n}$ is the normal force, and $\mathbf{f}_{i y}$ and $\mathbf{f}_{i z}$ are the shear force components.
Define $\mathbf{F}_{i n}, \mathbf{F}_{i y}, \mathbf{F}_{i z}$ as constant vectors along the normal and two shear directions on mesh $i$, we can express the dimensional force components using the non-dimensional force components $f_{i n}, f_{i y}, f_{i z}$ as $\mathbf{f}_{i k}=f_{i k} \mathbf{F}_{i k}, \quad k=n, y, z$. The total contact force is:

$$
\mathbf{f}_{i}=\sum_{k=n, y, z} f_{i k} \mathbf{F}_{i k}
$$

Similarly, defining $\mathbf{P}_{x}, \mathbf{P}_{y}, \mathbf{P}_{z}$ as constant vectors along the 3 axes of the world frame, $\mathbf{p}_{i}^{b}$ can be expressed as:

$$
\mathbf{p}_{i}^{b}=\sum_{j=x, y, z} p_{i j}^{b} \mathbf{P}_{j}
$$

Both position and force characteristic quantities are chosen such that $p_{i j}^{b} \in[-1,1], f_{i k} \in[-1,1]$.

Also note that equation (3) and (4) non-dimensionalized the force and position variables $\mathbf{f}_{i}$ and $\mathbf{p}_{i}^{b}$ into quantities $f_{i k}$ and $p_{i j}^{b}$. This is suggested before utilizing certain mathematical operations to avoid unit mismatch as seen in [17].

Having set up the contact positions and forces, whole body constraints can be imposed. The robot motion is assumed to be quasi-static, thus the robot is always subject to the static equilibrium constraint:

$$
\begin{gathered}
\sum_{i=1}^{N} \mathbf{f}_{i}+\mathbf{F}=0 \\
\sum_{i=1}^{N} \mathbf{p}_{i}^{b} \times \mathbf{f}_{i}+\mathbf{M}=0
\end{gathered}
$$

where $\mathbf{F}$ and $\mathbf{M}$ are known external forces (gravity $\mathbf{G}$ in this work) and moments. COM is also assumed to always be at the geometric center, irrespective of the robot limb motion.

Equation (6) introduces a bilinear term $\mathbf{p}_{i}^{b} \times \mathbf{f}_{i}$. Utilizing (3) and (4), the bilinear term can further be expressed as:

$$
\mathbf{p}_{i}^{b} \times \mathbf{f}_{i}=\sum_{j=x, y, z} \sum_{k=n, y, z} p_{i j}^{b} f_{i k} \mathbf{P}_{j} \times \mathbf{F}_{i k}
$$

To isolate the bilinear terms, let the moment variables be:

$$
m_{i j k}=p_{i j}^{b} f_{i k}
$$

where $m_{i j k}$ 's are the moment components. Plugging (8) into (7), and further back into (6) results in:

$$
\sum_{i=1}^{N} \sum_{j=x, y, z} \sum_{k=n, y, z} m_{i j k} \mathbf{P}_{j} \times \mathbf{F}_{i k}+\mathbf{M}=0
$$

In the predominant case where legged robots are not equipped with grippers on its toes, the point contacts with the environment are pure frictional contacts. When the robot places its toes, the contact forces are subject to friction cone constraints. Given (3) and defining $\mu$ as the friction coefficient, the constraint can be written as:

$$
\sqrt{f_{i y}^{2}+f_{i z}^{2}}<\mu f_{i n}
$$


To plan a complete quasi-static motion to the specified goal, the motion planner generates a series of "key frames"-body COM positions, body orientations, and footstep positions-to the goal position. The number of key frames is pre-specified as $M$, and each key frame posture needs to satisfy kinematics constraints. Between two consecutive key frames, step size constraints are enforced. Similar to [3], the following constraints are used:

$$
\begin{aligned}
& \text { for } r=1, \ldots, M \text {, } \\
& \Delta \underline{\mathbf{p}}_{\min } \leq\left\|\underline{\mathbf{p}}_{C O M}[r]-\underline{\mathbf{p}}_{C O M}[r-1]\right\|_{2} \leq \Delta \underline{\mathbf{p}}_{\max } \\
& \Delta \underline{\mathbf{P}}_{\min } \leq\left\|\underline{\mathbf{p}}_{i}[r]-\underline{\mathbf{p}}_{i}[r-1]\right\|_{2} \leq \Delta \underline{\mathbf{P}}_{\max } \\
& \Delta \underline{\Theta}_{\min } \leq\left\|\underline{\Theta}_{b}[r]-\underline{\Theta}_{b}[r-1]\right\|_{2} \leq \Delta \underline{\Theta}_{\max } \\
& \left\|\underline{\mathbf{p}}_{i}[r]-\underline{\mathbf{p}}_{C O M}[r]-\mathbf{R}[r] \underline{v}\right\|_{2} \leq \Delta_{F K}
\end{aligned}
$$

where $\Theta_{b}[r]$ is body orientation, $\Delta \underline{\mathbf{p}}_{\text {min }}, \Delta \underline{\mathbf{p}}_{\text {max }}, \Delta \underline{\mathbf{P}}_{\text {min }}$, $\Delta \underline{\mathbf{P}}_{\max }, \Delta \underline{\Theta}_{\min }$, and $\Delta \underline{\Theta}_{\max }$ are bounds for the toe, body $\mathrm{COM}$, and orientation step sizes. The limb workspace is simplified into a sphere, with $\Delta_{F K} \in \mathbb{R}$ its radius. $\underline{v}$ is the constant shoulder vector from body COM to the first joint of the limb (depicted in Fig. 2). $\mathbf{R}[r]$ is the rotation matrix as a function of $\underline{\Theta}_{b}[r]$. We assume the body rotation angles are small $(<15$ degrees), thus the rotation matrix can be linearized in terms of $\underline{\Theta}_{b}[r]=[\alpha, \beta, \gamma]$ [3].

In summary, there are kinematics constraints (11) (convex), (1) (2) (4) (linear), static equilibrium constraints (3) (5) (9) (linear), with additional constraints (8) (bilinear), and friction cone constraints (10) (convex). The objective function minimizes the distance of the planned final configuration to the goal configuration and penalizes the step size in similar fashion as [1][3]. Let us denote the complete problem by $P$. This problem is parametrized by $\theta$ which is the terrain geometry $\mathbf{v}_{i u}$. Fig. 2 illustrates the scene and the notations that are used in this paper. For simplicity, the decision variables are grouped into two sets - kinematics variables $\Gamma_{p}=\left\{\underline{\mathbf{p}}_{i}[r], p_{i j}[r], \underline{\mathbf{p}}_{C O M}[r], \underline{\Theta}_{b}[r]\right\}$ and force variables $\Gamma_{f}=\left\{\overline{\mathbf{f}}_{i}[r], f_{i k}[r], m_{i j k}[r]\right\}$.

A standard approach to convert bilinear constraints into MICPs is to grid the variable space (shown in Fig. 1 with $F_{1}$ ) and use McCormick envelopes to approximate the nonlinear constraint inside each grid. This approach accurately describes solutions for any problem parameter $\theta$, but introduces hundreds of binary variables into the optimization problem [11], hence solving speed is slow. In this paper, we solve problem $P$ by introducing a 2 -stage convex optimization process $P_{1} \rightarrow P_{2}$. During the first stage $P_{1}$, we approximate the bilinear constraints in $P_{2}$ using McCormick envelopes and solve for both sets of bilinear variables $p_{i j}$ and $f_{i k}$. In the second stage $P_{2}$, we choose to keep one set of bilinear variables in the solution of $P_{1}$ and project the other set onto the bilinear surface. If one set of bilinear variables are given, the bilinear constraint $(8)$ in $P_{2}$ is linearized. Because of the McCormick envelope in $P_{1}$, the 2-stage planner is coupled, with the approximation of the nonlinear constraint in $P_{2}$ embedded in $P_{1}$. This process is shown in Fig. 3

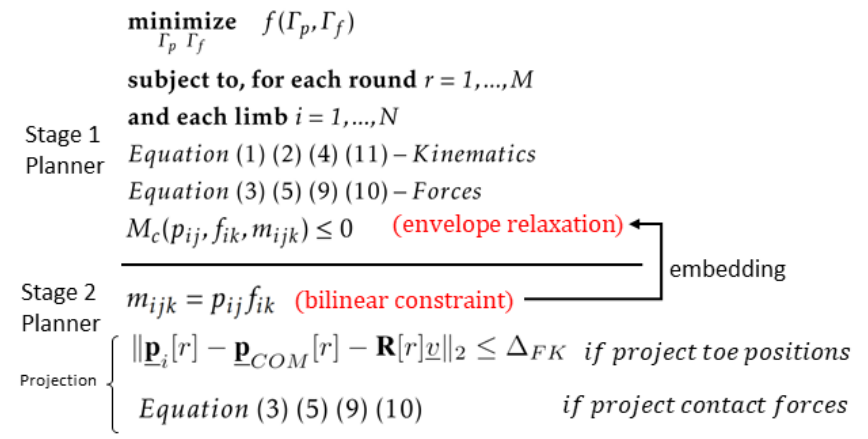

Fig. 3: Optimization formulation of the motion planning problem

We propose to learn the best envelope to a certain type of problem, such that the envelope formulation captures the heuristics behind the given type of input. Since trajectory optimization solves a series of postures with identical mathematical formulation, it is sufficient to learn the constraints on a single posture. We generate data by solving the problem with $M=1$ using the accurate MICP formulation [11], and collect data for bilinear variables. We then fit McCormick envelopes around the regions where data clusters, excluding the infeasible regions. Through this approach, the relaxation can be made tighter with a fixed number of envelopes.

\section{LEARNING SETUP}

We present the mathematical setup for the learning problem. As solutions of similar types of problems could be densely populated in certain regions, learning methods could be used to identify those regions to form a "tighter" McCormick envelope. This eliminates regions in the variables' space where infeasible solutions are expected.

Our algorithm seeks the overlapping regions for bilinear variables $(\mathbf{p}, \mathbf{f})$. The position variable $\mathbf{p}$ to be learned is $\mathbf{p}^{b}$. We omit the superscript for simplicity. Suppose we define problem $P\left(\theta_{i}\right)$ where $\theta_{i}$ is the parameter drawn from a distribution $G(\theta)$ and represents the terrain shape in our work. If $P\left(\theta_{i}\right)$ is feasible, we define the solution set $X\left(\theta_{i}\right)=\left\{\mathbf{x} \mid \mathbf{x}=(\mathbf{p}, \mathbf{f}, \mathbf{m}, \mathbf{y})\right.$ feasible for $\left.P\left(\theta_{i}\right)\right\}$. $\mathbf{p}, \mathbf{f}, \mathbf{m}$ are the variables that satisfy the bilinear constraint $m_{d}=$ $p_{d} f_{d}$ in each dimension $d=1, \ldots, D$, while $\mathbf{y}$ 's are other optimization variables. If $P\left(\theta_{i}\right)$ is infeasible, we define the infeasible solution set $\bar{X}\left(\theta_{i}\right)$ by removing the bilinear constraint from $P\left(\theta_{i}\right)$ that generates $\bar{P}\left(\theta_{i}\right)$, and $\bar{X}\left(\theta_{i}\right)=$ $\left\{\mathbf{x} \mid \mathbf{x}=(\mathbf{p}, \mathbf{f}, \mathbf{m}, \mathbf{y})\right.$ feasible for $\left.\bar{P}\left(\theta_{i}\right)\right\}$. A McCormick envelope relaxation of $m_{d}=p_{d} f_{d}$ [18] can be defined tightly over a pair of lower/upper bounds $\left[\mathbf{p}_{d}^{L}, \mathbf{p}_{d}^{U}\right]$ and $\left[\mathbf{f}_{d}^{L}, \mathbf{f}_{d}^{U}\right]$ that describes a rectangular region $\mathbf{B}$ over $\left[\mathbf{p}^{T}, \mathbf{f}^{T}\right]^{T}$. McCormick envelopes $M_{c}(\mathbf{B})$ satisfy the property such that $\left[\mathbf{p}^{T}, \mathbf{f}^{T}\right]^{T} \in$ $\mathbf{B}$ and $m_{d}=p_{d} f_{d}, \forall d$ implies $M_{c}(\mathbf{B}) \leq 0$. Furthermore, $\left[\mathbf{p}^{T}, \mathbf{f}^{T}\right]^{T} \notin \mathbf{B} \Rightarrow M_{c}(\mathbf{B}) \not \leq 0$. Define $S_{i}$ as the projection of $X\left(\theta_{i}\right)$ onto $\mathbf{p} \times \mathbf{f}$ (" $\times$ " is Cartesian product) subspace giving all feasible $\left[\mathbf{p}^{T}, \mathbf{f}^{T}\right]^{T}$ for problem $P\left(\theta_{i}\right)$. Then, $\forall(\mathbf{p}, \mathbf{f}) \in S_{i}$, $\exists(\mathbf{m}, \mathbf{y})$ such that $(\mathbf{p}, \mathbf{f}, \mathbf{m}, \mathbf{y})$ is feasible for $P\left(\theta_{i}\right)$. We also define the projection of infeasible solution set of $\left[\mathbf{p}^{T}, \mathbf{f}^{T}\right]^{T}$ as $\bar{S}_{i}$ by projecting $\bar{X}\left(\theta_{i}\right)$ onto $\mathbf{p} \times \mathbf{f}$ subspace.

Assume that for any feasible $P\left(\theta_{i}\right)$ that generates $S_{i}$, there exists an overlapping region: $\mathcal{S}=\cap S_{i} \neq \emptyset$. We can find a 
rectangular region $\mathbf{B}_{\mathcal{S}}$ inside $\mathcal{S}$ to construct a McCormick envelope relaxation $M_{c}\left(\mathbf{B}_{\mathcal{S}}\right) \leq 0$, and define the 2-step optimization process $P_{1} \rightarrow P_{2} . P_{1}$ is defined by replacing the biliner constraints in $P\left(\theta_{i}\right)$ with the McCormick relaxation, and gives a solution $(\widetilde{\mathbf{p}}, \widetilde{\mathbf{f}}, \widetilde{\mathbf{m}}, \widetilde{\mathbf{y}}) . P_{2}$ has identical constraints as $P$, but uses $(\widetilde{\mathbf{f}}, \widetilde{\mathbf{y}})$ (or $(\widetilde{\mathbf{p}}, \widetilde{\mathbf{y}}))$ from $P_{1}$ to solve for $\left(\mathbf{p}^{*}, \mathbf{m}^{*}\right)$ (or $\left(\mathbf{f}^{*}, \mathbf{m}^{*}\right)$ ), as the bilinear constraints are linearized.

Theorem. If there exist a McCormick envelope $M_{c}\left(\boldsymbol{B}_{\mathcal{S}}\right) \leq 0$ that satisfies the following 3 conditions:

1) $\forall \theta_{i}$ that makes $P\left(\theta_{i}\right)$ feasible and generates $S_{i}, \boldsymbol{B}_{\mathcal{S}} \cap$ $S_{i} \neq \emptyset$.

2) $\forall \theta_{i}$ that makes $P\left(\theta_{i}\right)$ infeasible, $\boldsymbol{B}_{\mathcal{S}} \cap \bar{S}_{i}=\emptyset$.

3) (Complete Projectability) $\forall \boldsymbol{f} \in \boldsymbol{B}_{\mathcal{S}}, \forall \boldsymbol{y}$, (or $\forall \boldsymbol{p} \in \boldsymbol{B}_{\mathcal{S}}$, $\forall \boldsymbol{y})$ such that $(\boldsymbol{f}, \boldsymbol{y})($ or $(\boldsymbol{p}, \boldsymbol{y}))$ is feasible for $P_{1},(\boldsymbol{f}, \boldsymbol{y})$ $($ or $(\boldsymbol{p}, \boldsymbol{y}))$ is also feasible for $P$.

Then the 2-step process $P_{1} \rightarrow P_{2}$ defined above satisfy:

1) $P$ is feasible $\Rightarrow P_{1} \rightarrow P_{2}$ is feasible. In addition, any feasible solution for $P_{1} \rightarrow P_{2}$ is also feasible for $P$.

2) $P$ is infeasible $\Rightarrow P_{1}$ is infeasible.

Note that depending on how $P_{2}$ projects the solution of $P_{1}$, the required projectability condition (3) is different. If we keep $(\mathbf{f}, \mathbf{y})$ and project $(\mathbf{p}, \mathbf{m})$, then we need $(\mathbf{f}, \mathbf{y})$ to be feasible for $P$, and vise versa.

\section{Proof.}

1) Suppose $P$ is feasible with solution subspace $S_{i}$. Since $\mathbf{B}_{\mathcal{S}} \cap S_{i} \neq \emptyset, \exists\left[\mathbf{p}_{i}^{T}, \mathbf{f}_{i}^{T}\right]^{T} \in \mathbf{B}_{\mathcal{S}} \cap S_{i} .\left[\mathbf{p}_{i}^{T}, \mathbf{f}_{i}^{T}\right]^{T} \in S_{i} \Rightarrow$ $\exists\left(\mathbf{m}_{i}, \mathbf{y}_{i}\right)$ such that $\mathbf{x}=\left(\mathbf{p}_{i}, \mathbf{f}_{i}, \mathbf{m}_{i}, \mathbf{y}_{i}\right)$ is feasible for $P$ (satisfying each constraint except $M_{c}\left(\mathbf{p}_{i}, \mathbf{f}_{i}, \mathbf{m}_{i}\right) \leq 0$ ). In particular, $\left(\mathbf{p}_{i}, \mathbf{f}_{i}, \mathbf{m}_{i}\right)$ satisfies the bilinear constraint $m_{d}=p_{d} f_{d}, \forall d$. This together with $\left[\mathbf{p}_{i}^{T}, \mathbf{f}_{i}^{T}\right]^{T} \in \mathbf{B}_{\mathcal{S}}$ implies $M_{c}\left(\mathbf{p}_{i}, \mathbf{f}_{i}, \mathbf{m}_{i}\right) \leq 0$. Thus $P$ is feasible $\Rightarrow P_{1}$ is feasible.

Now we show that $P_{1}$ is feasible $\Rightarrow P_{2}$ is feasible, and the solution of $P_{1} \rightarrow P_{2}$ is feasible for $P$. Any feasible solution $(\mathbf{p}, \mathbf{f}, \mathbf{m}, \mathbf{y})$ for $P_{1}$ satisfies $M_{c}(\mathbf{p}, \mathbf{f}, \mathbf{m}) \leq 0 \Rightarrow(\mathbf{p}, \mathbf{f}) \in \mathbf{B}_{\mathcal{S}}$. By condition (3), $(\mathbf{f}, \mathbf{y})$ (or $(\mathbf{p}, \mathbf{y})$ ) is feasible for $P$, which means $\exists(\widetilde{\mathbf{p}}, \widetilde{\mathbf{m}})$ (or $(\widetilde{\mathbf{f}}, \widetilde{\mathbf{m}})$ ) such that $\widetilde{\mathbf{x}}=(\widetilde{\mathbf{p}}, \mathbf{f}, \widetilde{\mathbf{m}}, \mathbf{y})$ (or $(\mathbf{p}, \widetilde{\mathbf{f}}, \widetilde{\mathbf{m}}, \mathbf{y}))$ satisfies $P$. Since $P_{2}$ has identical constraints as $P, \widetilde{\mathbf{x}}$ is feasible for $P_{2}$. As $P_{2}$ is feasible and has identical constraints as $P$, any solution for $P_{2}$ is feasible for $P$.

2) By condition (2), $\mathbf{B}_{\mathcal{S}} \cap \bar{S}_{i}=\emptyset, \forall\left[\mathbf{p}^{T}, \mathbf{f}^{T}\right]^{T} \in \bar{S}_{i}$ (satisfies all but the bilinear constraints), $\left[\mathbf{p}^{T}, \mathbf{f}^{T}\right]^{T} \notin \mathbf{B}_{\mathcal{S}}$, thus $M_{c}(\mathbf{p}, \mathbf{f}, \forall \mathbf{m}) \not \leq 0 \Rightarrow P_{1}$ is infeasible.

Remark. It may look like result 2) is too strong. Normally, a relaxation is feasible doesn't guarantee that the original problem is feasible. However, in this case it does. Basically, 2 ) shifts the region of relaxation on $(\boldsymbol{p}, \boldsymbol{f})$ completely away from the danger zone - region that potentially makes infeasible problems feasible. The relaxation will admit new $\boldsymbol{m}$ points. However, no matter what $\boldsymbol{m}$ is, since $(\boldsymbol{p}, \boldsymbol{f})$ is infeasible for the original problem, $(\boldsymbol{p}, \boldsymbol{f}, \boldsymbol{m}, \boldsymbol{y})$ wll not be feasible. A good example is that, for some problem, $\boldsymbol{m}=\boldsymbol{p} \boldsymbol{f}$ does not constraint anything. This means whenever $\theta_{i}$ is infeasible for $P, \bar{P}$ is still infeasible. In this case, the envelope can be all

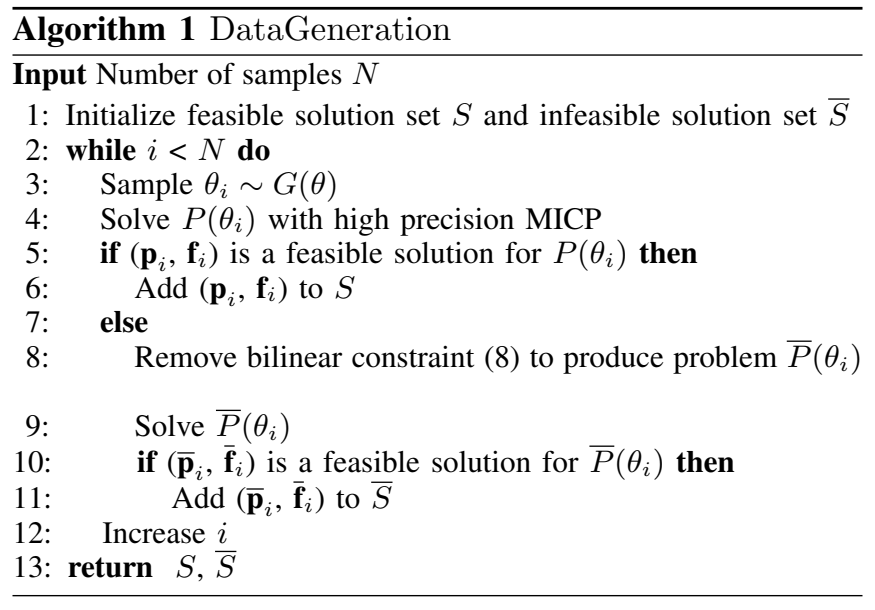

the domain, as other constraints for $P$ are already making the problem infeasible.

The idea behind finding $\mathbf{B}_{\mathcal{S}}$ is shown in Fig. 1 Condition (3) guarantees projectability for any point in $\mathbf{B}_{\mathcal{S}}$. Strictly satisfying it guarantees $P_{1}$ will not give solutions that cause $P_{2}$ to be infeasible. In practice, verifying condition (3) is very difficult. We put additional safety factors to make constraints in $P_{1}$ even tighter to reduce the the relaxation (at the risk of $P_{1}$ not a complete relaxation of $P$ ). As an simple extension of this paper, one can also learn an overlapping region of $y_{i}$ 's and formulate as an additional constraint into $P_{1}$. This guarantees that $P_{1}$ does not give any "strange" $y_{i}$ that makes $P_{2}$ infeasible. Another approach is to set an optimal criteria for $P_{1}$ and guarantee that the optimal solution of $(\mathbf{f}, \mathbf{y})$ is always projectable to $P_{2}$ [19]. If the projection still fails with the above efforts, the problem is over-relaxed, suggesting that the envelope should be divided into multiple smaller pieces. Interesting future work remains where an objective function that guarantees projectability at optimal points could potentially exist. In addition, if we do not confine the formulation to convex ones, we can use nonlinear optimization (NLPs) in $P_{2}$ to do the projection. In this case, we only keep $y_{i}$ from $P_{1}$ and project $(\mathbf{p}, \mathbf{y})$ simultaneously. Properly initialized NLPs can have fast speed with a solvable rate close to $100 \%$ [11]. Finally, even if $\cap S_{i}=\emptyset$, we can still identify multiple mutually exclusive $\mathbf{B}_{\mathcal{S}}$ 's that give multiple envelopes. An example is shown in the next section.

We present two data-based approaches to identify $\mathbf{B}_{\mathcal{S}}$, one based on clustering to directly fit envelopes and the other one based on evolutionary algorithms.

1) Clustering Approach: Based on the high-accuracy MICP formulation [11], we can generate feasible solutions or prove infeasibility for problems $P\left(\theta_{i}\right)$ sampled from $G(\theta)$. We recognize that if we sample the same amount of feasible solutions inside each $S_{i}$, the overlapping region $\mathcal{S}$ will receive more samples, indicating a clustering approach may identify $\mathcal{S}$. We also need to draw samples from the infeasible solution set $\bar{S}_{i}$ and make the envelope exclude those points. The data generation algorithm is shown in Algorithm 1, where we generate a random terrain from a predefined distribution and collect the subsequent optimization's 


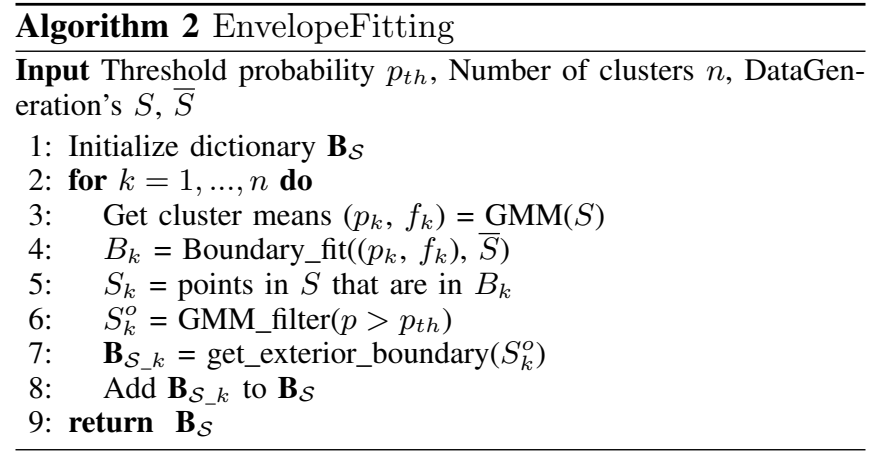

solutions depending on their feasibilities.

Having collected the data in $S$ and $\bar{S}$, we use Algorithm 2 to fit the envelopes. With a specified number of envelopes, the algorithm first performs clustering with Gaussian Mixture Models (GMMs) to identify the center of clusters. Then, an optimization problem named Boundary_fit is solved to fit the largest rectangular region $B$ around the centers excluding any points in $\bar{S}_{i}$. Multiple formulations can be used to achieve this. We used a formulation based on mixed-integer programming, but other options exist [20]. We then collect all points in $B$ but remove those whose probability of belonging to the cluster is less than the threshold $p_{t h}$. The exterior boundary formed by the remaining points gives $\mathbf{B}_{\mathcal{S}}$.

2) Evolutionary Approach: Contrasting to the fitting approach, a sampling-based evolutionary approach could, by nature, find bounds that are even tighter. Using a sufficient number of random terrain (input) and feasibility (output) pairs, a genetic algorithm (GA) can be tailored to solve a bilevel optimization that is indicative of the original problem at hand. While mostly following the conventional GA approach, we choose our chromosomes to be the lower and upper bounds of the envelope, while uniform crossover is done per lower/upper bound pair as opposed to per gene value. We design the fitness function to be representative of the bilevel optimization that occurs between the two stages of the original problem. To achieve this, we define two key metrics that help in finding better envelopes. We define $a$ to be the percentage of original infeasible values becoming feasible, and $b$ to be the percentage of original feasible values becoming infeasible. At each solution's fitness calculation, a random pair of terrain parameter and ground truth feasibility $z_{g}$ are selected from a pre-generated dataset built using the data generator. Then, the individual and the terrain parameter are used to find the feasibility $z_{1}$ in $P_{1}$. If $z_{g}$ is infeasible while $z_{1}$ is feasible, $a$ increases, whereas if $z_{g}$ is feasible but $z_{1}$ is infeasible, $b$ increases. Per generation, each individual is tested against $K$ number of terrains from the training set and the average $a$ and $b$ are used in the calculation of the fitness function. While we try to minimize $a$, we keep $b$ below a certain threshold $\delta$. To ensure that the number of mutations also decreases over the generations and that the variance of the population's fitness decreases, the mutation rate is set to be a function of the generation number decreasing over time.

\section{EXPERIMENT}

\section{A. Training}

To validate our proposed algorithm, we choose the random distribution $\theta$ to provide two different kinds of terrainsground and wall-whose $(\mathbf{p}, \mathbf{f})$ are expected to show distinct distributions. For training, we provide one terrain mesh to each leg, while varying terrain position, orientation, and friction coefficient $\mu$ randomly. For ground data, we uniformly sample the angle of normal vectors within the $30^{\circ}$ region around the straight-up direction, while varying $\mu$ between $[0.1,0.8]$. The wall data are collected to plan trajectories for the robot to climb up between two walls [21] with pure frictional contact. We vary the angles within the $30^{\circ}$ region around the nominal direction as shown in the top right of Fig. 5, and vary $\mu$ between $[0.1,1.2]$. We use Algorithm 1 to gather a set $S$ of 500 feasible points and 500 infeasible points for envelope fitting, and another set $\bar{S}$ of the same number for validation. We set the number of envelopes to be 1 . Both ground and wall envelopes are fit with 3 legs (two right, one left). Envelopes are also fitted with 5 legs on the wall. For validation, we separate the success rate into two categories showing respectively if our convex optimization can identify feasible solutions correctly and identify infeasible solutions correctly. We show the results of both stages. If $\mathbf{B}_{\mathcal{S}} \in S_{i}, P_{1}$ should remain feasible if $P$ is feasible. If condition (2) in our theorem holds, $P_{1}$ should be infeasible if $P$ is infeasible. If condition (3) holds, the feasible solutions for $P_{1}$ should be projectable to make $P_{2}$ feasible, thus success rate for feasible solutions should not drop from $P_{1}$ to $P_{2}$. We use both convex and NLP methods mentioned in the previous section for $P_{2}$. For convex method, we use $\mathbf{p}^{b}$ from $P_{1}$ to solve $\mathbf{f}$ in $P_{2}$. The NLP method solves $\mathbf{p}^{b}$ and $\mathbf{f}$ together.

Expert human heuristics are used to create envelope parameters as baselines. Bounds for $\mathbf{p}^{b}$ are measured outer boundaries of each leg's workspace, while that for $\mathbf{f}$ are from our understanding of force profiles. Normal force bounds are $[0.0,0.9] /[0.0,0.5]$ for climbing/walking, as large normal forces are expected to prevent slipping. Shear force bounds are $[-0.3,0.3] /[-0.15,0.15]$ for climbing/walking. For wall climbing the vertical shear force boundeds are $[0.0,0.4]$ as they need to counteract the gravity. We perform hand-tuning to optimize the performance. The results are in Table [1]

The results show that $P_{1}$ tends to match $P$ well for all test cases when using clustering, as both success rates are close to $100 \%$. For convex projection, about $20 \%$ 30\% of $P_{1}$ 's solutions cannot be projected for 3 leg case. This is due to a violation of condition (3) indicating a single envelope may over-relax the original problem. For the 5 leg test, both stages perform well. Intuitively, since the problem dimension is higher, $\mathbf{p}$ has more room to adjust at $P_{2}$. NLP projections perform well, with the rate of projection close to $100 \%$ and solving speed no more than a few hundred milliseconds.

Aside from training a single cluster, we tried to fit envelopes with combined ground and wall data. By giving $n=2$ in Algorithm 2, two mutually exclusive envelopes representing the ground and the wall are identified. This 
TABLE I: Validation results for trained envelopes

\begin{tabular}{|c|c|c|c|c|c|}
\hline \multirow{2}{*}{\multicolumn{2}{|c|}{ Problem }} & \multicolumn{2}{|c|}{$P_{1}$} & \multirow{2}{*}{$\begin{array}{l}P_{1 \rightarrow P_{2}} \\
\text { correct } \\
\text { feasible* }\end{array}$} & \multirow{2}{*}{$\begin{array}{c}P_{1} \rightarrow P_{2}(\mathrm{NLP}) \\
\text { correct } \\
\text { feasible }\end{array}$} \\
\hline & & correct & correct & & \\
\hline \multirow{3}{*}{$\begin{array}{l}3 \mathrm{leg} \\
\text { ground }\end{array}$} & Cluster & $98.16 \%$ & $77.78 \%$ & $83.54 \%$ & $98.16 \%$ \\
\hline & GA & $70.08 \%$ & $91.24 \%$ & $37.40 \%$ & $70.08 \%$ \\
\hline & Heuristic & $99.77 \%$ & $50.00 \%$ & $84.91 \%$ & $99.77 \%$ \\
\hline \multirow{3}{*}{$\begin{array}{l}3 \text { leg } \\
\text { wall }\end{array}$} & Cluster & $93.62 \%$ & $80.00 \%$ & $63.32 \%$ & $93.62 \%$ \\
\hline & GA & $77.85 \%$ & $97.72 \%$ & $67.07 \%$ & $77.85 \%$ \\
\hline & Heuristic & $78.72 \%$ & $51.33 \%$ & $60.75 \%$ & $78.72 \%$ \\
\hline \multirow{3}{*}{$\begin{array}{l}5 \text { leg } \\
\text { wall }\end{array}$} & Cluster & $95.27 \%$ & $79.31 \%$ & $90.41 \%$ & $93.49 \%$ \\
\hline & GA & $68.21 \%$ & $98.68 \%$ & $62.70 \%$ & $68.21 \%$ \\
\hline & Heuristic & $92.90 \%$ & $72.41 \%$ & $88.10 \%$ & $92.25 \%$ \\
\hline
\end{tabular}

* Correct infeasible results for $P_{2}$ are identical to $P_{1}$ thus omitted.

results in an MICP formulation with one binary variable $z \in\{0,1\}$ per posture that switches between 2 modes as the robot traverses from one type of terrain to the other.

Contrary to the clustering approach, the GA approach shows lopsided results. This could be an artifact of suboptimal initial solutions and a lack of generations as seen by the variance in Fig. 4 However, the extremely high infeasibility detection suggests that possibly a combined approach between a structured clustering and a conventional learning based approach could achieve higher accuracies.

The results suggest that our methodological approach based on data immediately provides comparable results to heuristics that require expert knowledge.

\section{B. Planning}

We use the learned envelopes in our 2-stage planner, and plan the motion for a 24 DoF hexapod. Trajectories to traverse in multiple terrains are found, including walking on flat ground with stairs, climbing between two flat vertical walls of varying friction, and climbing inside a tube. The coupling achieved in the planner is compared against the decoupled approaches [3]. The planner is verified on hardware. All results are included in the accompanying video [22].

1) Walking on Ground: The proposed planner generates trajectories for walking on a flat ground with stairs (Fig. 5 top left). This shows the feasibility of the approach on a simple environment because the terrains are effectively flat.

2) Climbing between Walls: This problem is first studied by [21], which uses a stiffness based approach allowing the robot to brace between two walls and climb. A 2-stage decoupled approach is then used [3] to plan the climbing motion, where it plans the position p's without any knowledge of force. To test the proposed coupled planner, we plan the trajectory for the robot to climb on the walls with patches of zero friction $\mu=0$ (green regions in Fig. 5, bottom right) and $\mu=1$ in other areas. $P_{1}$ will have to place the toes outside the region to avoid $P_{2}$ being infeasible. For this, decoupled planner [3] would fail. We also plan a climbing motion inside a tube consisting of meshes at different angles (Fig. 5 , bottom
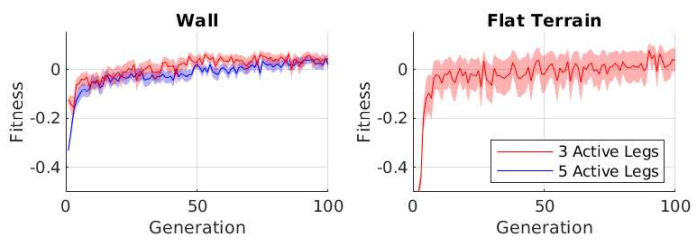

Fig. 4: The average fitness of the population is shown with its variance. Certain individuals maximize the fitness early on in the generation.

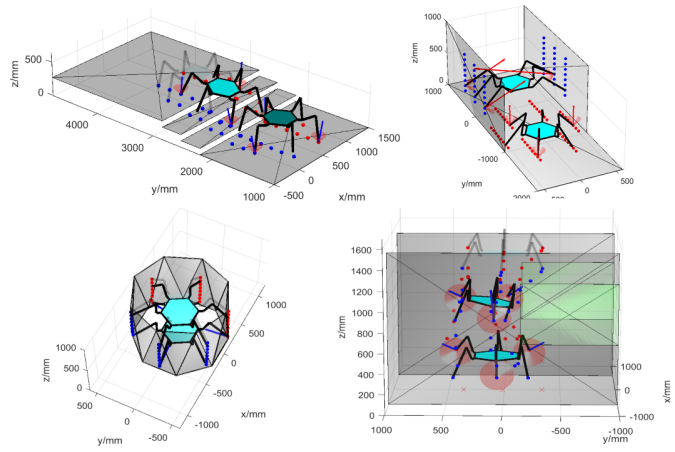

Fig. 5: Motion plans generated by the proposed planner. Top left: the robot climbs stairs. Top right: the robot switches from walking to climbing. Bottom left: the robot climbs up inside a tube. Bottom right: the robot climbs up between two walls and avoid patches of low friction materials.

TABLE II: Solving time for vertical two flat wall climbing

\begin{tabular}{ccccc}
\hline Test Name & Rounds $M$ & Variables & Constraint & Solve Time $[\mathbf{s}]^{*}$ \\
\hline \hline 2-stage Convex & 8 & 4128 & 1176 & 0.16 \\
\hline \hline MICP without Envelope & 4 & $16608(276$ binary $)$ & 3360 & 91 \\
\hline MICP with Envelope & 4 & $16608(276$ binary $)$ & 4008 & 33 \\
\hline \hline MICP without Envelope & 8 & $33216(552$ binary $)$ & 6720 & $>1,000$ \\
\hline MICP with Envelope & 8 & $33216(552$ binary $)$ & 8016 & 152 \\
\hline
\end{tabular}

* Data taken on an Intel i5-6260U 1.80GHz machine with Gurobi [23]

left), to test the adaptability to irregular walls. In both cases, the planer solves feasible motion plan on hardware.

3) Automatic switching motion plan from ground to wall: We demonstrate that with the learned 2 mode formulation presented in Section IV-A, trajectories with contact forces that automatically switch between walking and climbing can be generated as seen in Fig. 5 top right. Beyond finding a feasible trajectory, the planner finds $z=0 / z=1$ when the robot is on the ground/wall, indicating that it interprets the current motion as walking/climbing. This result shows the interpretability of the proposed planner. If we divide the variable space into smaller pieces and assign an integer variable for each piece, the information found by the planner can quickly get submerged by the large number of possible combinations of integer variables. Instead, our planner gives interpretable information that humans can process.

4) Solving Time: We benchmark the solving time on the problem of climbing between two flat walls with uniform $\mu$. Table II row 1 shows the solving time for the proposed twostage convex planner. The convex solver generates trajectories in hundreds of milliseconds. Comparison with similar works using NLPs suggests a possible speed up of around 100 times [2]. This justifies the motivation behind using a multi-staged convex algorithm. The designed envelope can also be used to speed up MICP. We compare the accurate MICP formulation with and without our learned envelope as an additional constraint for the problem of climbing between two flat walls. A typical MICP solver deals with integer variables through a branch and bound algorithm [24] that expands nodes on each binary variable. Since [11] does not utilize the problem-specific knowledge, the solver wastes time expanding nodes inside regions that we already know are infeasible. Our approach reduces the solving time, and has minimum impact on the solutions (Table III row 2-4). 


\section{COnclusion, Discussion And Future WORK}

We propose a 2-stage motion planner based on convex optimization, with the inter-stage coupling formulated as McCormick envelopes learned from data. We performed learning through clustering and GA approaches and validated against labeled data. The results show that a smaller number of integer variables and envelopes tailored to the type of problem can reduce solve time and help interpret the outcome. We also demonstrated the planner on the hardware.

Finer relaxations with smaller envelopes could be possible if one envelope is insufficient. Learning an objective function that guides the 1st stage solution to a projectable point is another future work. Since we require exploring the solution set, which could be efficient on hardware, training on hardware is of interest. While we focused on a specific problem, this work may be generalizable for more complicated problems with multiple stages, and a larger class of nonlinear constraints.

\section{REFERENCES}

[1] S. Kuindersma, R. Deits, M. Fallon, A. Valenzuela, H. Dai, F. Permenter, T. Koolen, P. Marion, and R. Tedrake, "Optimization-based locomotion planning, estimation, and control design for the atlas humanoid robot," Autonomous Robots, vol. 40, no. 3, pp. 429-455, 2016.

[2] A. W. Winkler, C. D. Bellicoso, M. Hutter, and J. Buchli, "Gait and trajectory optimization for legged systems through phase-based endeffector parameterization," IEEE Robotics and Automation Letters, vol. 3, no. 3, pp. 1560-1567, 2018.

[3] X. Lin, J. Zhang, J. Shen, G. Fernandez, and D. W. Hong, "Optimization based motion planning for multi-limbed vertical climbing robots," in 2019 IEEE/RSJ International Conference on Intelligent Robots and Systems (IROS). IEEE, 2019, pp. 1918-1925.

[4] M. S. Ahn, H. Chae, and D. W. Hong, "Stable, autonomous, unknown terrain locomotion for quadrupeds based on visual feedback and mixed-integer convex optimization," in 2018 IEEE/RSJ International Conference on Intelligent Robots and Systems (IROS). IEEE, 2018, pp. 3791-3798.

[5] B. Plancher and S. Kuindersma, "Realtime model predictive control using parallel ddp on a gpu," in Toward Online Optimal Control of Dynamic Robots Workshop at the 2019 International Conference on Robotics and Automation (ICRA), Montreal, Canada, 2019.

[6] P. Vernaza, M. Likhachev, S. Bhattacharya, S. Chitta, A. Kushleyev, and D. D. Lee, "Search-based planning for a legged robot over rough terrain," in 2009 IEEE International Conference on Robotics and Automation. IEEE, 2009, pp. 2380-2387.

[7] S. Boyd and L. Vandenberghe, Convex optimization. Cambridge university press, 2004.

[8] M. Kalakrishnan, J. Buchli, P. Pastor, M. Mistry, and S. Schaal, "Learning, planning, and control for quadruped locomotion over challenging terrain," The International Journal of Robotics Research, vol. 30, no. 2, pp. 236-258, 2011.

[9] M. Zucker, J. A. Bagnell, C. G. Atkeson, and J. Kuffner, "An optimization approach to rough terrain locomotion," in 2010 IEEE International Conference on Robotics and Automation. IEEE, 2010, pp. 3589-3595.

[10] G. P. McCormick, "Computability of global solutions to factorable nonconvex programs: Part i-convex underestimating problems," Mathematical programming, vol. 10, no. 1, pp. 147-175, 1976.

[11] H. Dai, G. Izatt, and R. Tedrake, "Global inverse kinematics via mixedinteger convex optimization," The International Journal of Robotics Research, vol. 38, no. 12-13, pp. 1420-1441, 2019.

[12] D. Bertsimas and B. Stellato, "The voice of optimization," Machine Learning, pp. 1-29, 2020.

[13] A. Cauligi, P. Culbertson, B. Stellato, D. Bertsimas, M. Schwager, and M. Pavone, "Learning mixed-integer convex optimization strategies for robot planning and control," arXiv preprint arXiv:2004.03736, 2020.
[14] A. M. Gleixner and S. Weltge, "Learning and propagating lagrangian variable bounds for mixed-integer nonlinear programming," in International Conference on AI and OR Techniques in Constriant Programming for Combinatorial Optimization Problems. Springer, 2013, pp. 355-361.

[15] H. Nagarajan, M. Lu, E. Yamangil, and R. Bent, "Tightening mccormick relaxations for nonlinear programs via dynamic multivariate partitioning," in International Conference on Principles and Practice of Constraint Programming. Springer, 2016, pp. 369-387.

[16] H. Nagarajan, M. Lu, S. Wang, R. Bent, and K. Sundar, "An adaptive, multivariate partitioning algorithm for global optimization of nonconvex programs," Journal of Global Optimization, vol. 74, no. 4, pp. 639-675, 2019.

[17] B. Ponton, A. Herzog, S. Schaal, and L. Righetti, "A convex model of humanoid momentum dynamics for multi-contact motion generation," in 2016 IEEE-RAS 16th International Conference on Humanoid Robots (Humanoids). IEEE, 2016, pp. 842-849.

[18] P. M. Castro, "Tightening piecewise mccormick relaxations for bilinear problems," Computers \& Chemical Engineering, vol. 72, pp. 300-311, 2015.

[19] B. Acikmese and S. R. Ploen, "Convex programming approach to powered descent guidance for mars landing," Journal of Guidance, Control, and Dynamics, vol. 30, no. 5, pp. 1353-1366, 2007.

[20] R. L. H. Deits, "Convex segmentation and mixed-integer footstep planning for a walking robot," Ph.D. dissertation, Massachusetts Institute of Technology, 2014.

[21] X. Lin, H. Krishnan, Y. Su, and D. W. Hong, "Multi-limbed robot vertical two wall climbing based on static indeterminacy modeling and feasibility region analysis," in 2018 IEEE/RSJ International Conference on Intelligent Robots and Systems (IROS). IEEE, 2018, pp. 4355-4362.

[22] RoMeLa. Icra2021 - multi-stage coupled convex programming with data-driven mccormick envelopes. YouTube. [Online]. Available: https://youtu.be/1WqTIVpFpIw

[23] G. Optimization, "Inc.,"gurobi optimizer reference manual," 2015," 2014.

[24] H. P. Williams, Model building in mathematical programming. John Wiley \& Sons, 2013. 